\title{
Oscillation Criteria for a Class of Second-Order Neutral Delay Dynamic Equations of Emden-Fowler Type
}

\author{
Zhenlai Han, ${ }^{1,2}$ Tongxing Li, ${ }^{1,2}$ Shurong Sun, ${ }^{1,3}$ Chao Zhang, ${ }^{1}$ \\ and Bangxian Han ${ }^{4}$
}

${ }^{1}$ School of Science, University of Jinan, Jinan, Shandong 250022, China

${ }^{2}$ School of Control Science and Engineering, Shandong University, Jinan, Shandong 250061, China

${ }^{3}$ Department of Mathematics and Statistics, Missouri University of Science and Technology Rolla, Missouri 65409-0020, USA

${ }^{4}$ Department of Mathematics, University of Science and Technology, Hefei 230026, China

Correspondence should be addressed to Zhenlai Han, hanzhenlai@163.com

Received 31 August 2010; Accepted 30 September 2010

Academic Editor: Elena Braverman

Copyright (C) 2011 Zhenlai Han et al. This is an open access article distributed under the Creative Commons Attribution License, which permits unrestricted use, distribution, and reproduction in any medium, provided the original work is properly cited.

We establish some new oscillation criteria for the second-order neutral delay dynamic equations of Emden-Fowler type, $\left[a(t)(x(t)+r(t) x(\tau(t)))^{\Delta}\right]^{\Delta}+p(t) x^{\gamma}(\delta(t))=0$, on a time scale unbounded above. Here $r>0$ is a quotient of odd positive integers with a and $p$ being real-valued positive functions defined on $\mathbb{T}$. Our results in this paper not only extend and improve the results in the literature but also correct an error in one of the references.

\section{Introduction}

The study of dynamic equations on time scales, which goes back to its founder Hilger [1], is an area of mathematics that has recently received a lot of attention. It was partly created in order to unify the study of differential and difference equations. Many results concerning differential equations are carried over quite easily to corresponding results for difference equations, while other results seem to be completely different from their continuous counterparts. The study of dynamic equations on time scales reveals such discrepancies and helps avoid proving results twice—once for differential equations and once again for difference equations.

The three most popular examples of calculus on time scales are differential calculus, difference calculus, and quantum calculus (see Kac and Cheung [2]), that is, when 
$\mathbb{T}=\mathbb{R}, \mathbb{T}=\mathbb{N}$, and $\mathbb{T}=q^{\mathbb{N}_{0}}=\left\{q^{t}: t \in \mathbb{N}_{0}\right\}$, where $q>1$. Many other interesting time scales exist, and they give rise to many applications (see [3]). Dynamic equations on a time scale have an enormous potential for applications such as in population dynamics. For example, it can model insect populations that are continuous while in season, die out in, for example, winter, while their eggs are incubating or dormant, and then hatch in a new season, giving rise to a nonoverlapping population (see [3]). There are applications of dynamic equations on time scales to quantum mechanics, electrical engineering, neural networks, heat transfer, and combinatorics. A recent cover story article in New Scientist [4] discusses several possible applications. Several authors have expounded on various aspects of this new theory; see the survey paper by Agarwal et al. [5] and references cited therein. A book on the subject of time scales, by Bohner and Peterson [3], summarizes and organizes much of time scale calculus; see also the book by Bohner and Peterson [6] for advances results of dynamic equations on time scales.

In recent years, there has been much research activity concerning the oscillation and nonoscillation of solutions of various dynamic equations on time scales unbounded above and neutral differential equations; we refer the reader to the papers [7-19]. Some authors are especially interested in obtaining sufficient conditions for the oscillation or nonoscillation of solutions of first and second-order linear and nonlinear neutral functional dynamic equations on time scales; we refer to the articles [20-28].

Agarwal et al. [7] considered the second-order delay dynamic equations

$$
x^{\Delta \Delta}(t)+p(t) x(\tau(t))=0, \quad t \in \mathbb{T}
$$

and established some sufficient conditions for oscillation of (1.1). Şahiner [11] studied the second-order nonlinear delay dynamic equations

$$
x^{\Delta \Delta}(t)+p(t) f(x(\tau(t)))=0, \quad t \in \mathbb{T}
$$

and obtained some sufficient conditions for oscillation by employing Riccati transformation technique. Zhang and Zhu [13] examined the second-order dynamic equations

$$
x^{\Delta \Delta}(t)+p(t) f(x(t-\tau))=0, \quad t \in \mathbb{T},
$$

and by using comparison theorems, they proved that oscillation of (1.3) is equivalent to the oscillation of the nonlinear dynamic equations

$$
x^{\Delta \Delta}(t)+p(t) f(x(\sigma(t)))=0, \quad t \in \mathbb{T}
$$

and established some sufficient conditions for oscillation by applying the results established in [15]. Erbe et al. [16] investigated the oscillation of the second-order nonlinear delay dynamic equations

$$
\left(r(t) x^{\Delta}(t)\right)^{\Delta}+p(t) f(x(\tau(t)))=0, \quad t \in \mathbb{T}
$$


and by employing the generalized Riccati technique, they established some new sufficient conditions which ensure that every solution of (1.5) oscillates or converges to zero. Mathsen et al. [20] investigated the first-order neutral delay dynamic equations

$$
[y(t)-r(t) y(\tau(t))]^{\Delta}+p(t) y(\delta(t))=0, \quad t \in \mathbb{T}
$$

and established some new oscillation criteria which as a special case involve some wellknown oscillation results for first-order neutral delay differential equations. Zhu and Wang [21] studied the nonoscillatory solutions to neutral dynamic equations

$$
[y(t)+p(t) y(g(t))]^{\Delta}+f(t, x(h(t)))=0, \quad t \in \mathbb{T}
$$

and gave a classification scheme for the eventually positive solutions of (1.7). Agarwal et al. [22], Şahíner [23], Saker et al. [24-26], Wu et al. [27], and Zhang and Wang [28] considered the second-order nonlinear neutral delay dynamic equations

$$
\left(r(t)\left((y(t)+p(t) y(\tau(t)))^{\Delta}\right)^{r}\right)^{\Delta}+f(t, y(\delta(t)))=0, \quad t \in \mathbb{T}
$$

where $\gamma>0$ is a quotient of odd positive integers, the delay function $\tau$ and $\delta$ satisfy $\tau: \mathbb{T} \rightarrow \mathbb{T}$ and $\delta: \mathbb{T} \rightarrow \mathbb{T}$ for all $t \in \mathbb{T}$, and $r$ and $p$ are real-valued positive functions defined on $\mathbb{T}$, and

$$
\left(h_{1}\right) r(t)>0, \int_{t_{0}}^{\infty}(1 / r(t))^{1 / \gamma} \Delta t=\infty \text {, and } 0 \leq p(t)<1 ;
$$

$\left(h_{2}\right) f: \mathbb{T} \times \mathbb{R} \rightarrow \mathbb{R}$ is continuous function such that $u f(u)>0$ for all $u \neq 0$, and there exists a nonnegative function $q$ defined on $\mathbb{T}$ such that $|f(t, u)| \geq q(t)|u|^{\gamma}$.

By employing different Riccati transformation technique, the authors established some oscillation criteria for all solutions of (1.8).

Recently, some authors have been interested in obtaining sufficient conditions for the oscillation and nonoscillation of solutions of Emden-Fowler type dynamic equations on time scales, differential equations, and difference equations; see, for example, [29-47].

Han et al. [32] studied the second-order Emden-Fowler delay dynamic equations

$$
x^{\Delta \Delta}(t)+p(t) x^{\gamma}(\tau(t))=0, \quad t \in \mathbb{T}
$$

and established some sufficient conditions for oscillation of (1.9) and extended the results given in [7].

Saker [34] studied the second-order superlinear neutral delay dynamic equation of Emden-Fowler type

$$
\left[a(t)(y(t)+r(t) y(\tau(t)))^{\Delta}\right]^{\Delta}+p(t)|y(\delta(t))|^{\gamma} \operatorname{sign} y(\delta(t))=0
$$

on a time scale $\mathbb{T}$. 
The author assumes that

$\left(A_{1}\right) \gamma>1$

$\left(A_{2}\right)$ the delay functions $\tau$ and $\delta$ satisfy $\tau: \mathbb{T} \rightarrow \mathbb{T}, \delta: \mathbb{T} \rightarrow \mathbb{T}, \tau(t) \leq t, \delta(t) \leq t$ for all $t \in \mathbb{T}$, and $\lim _{t \rightarrow \infty} \tau(t)=\lim _{t \rightarrow \infty} \delta(t)=\infty ;$

$\left(A_{3}\right) a, r$ and $p$ are positive rd-continuous functions defined on $\mathbb{T}$ such that $a^{\Delta}(t) \geq$ $0, \int_{t_{0}}^{\infty}(\Delta t / a(t))=\infty$, and $0 \leq r(t)<1$.

The main result for the oscillation of (1.10) in [34] is the following.

Theorem 1.1 (see, [34, Theorem 3.1]). Assume that $\left(A_{1}\right)-\left(A_{3}\right)$ hold. Furthermore, assume that

$$
\int_{t_{0}}^{\infty} p(t)(1-r(\delta(t)))^{\gamma} \delta^{\gamma}(t) \Delta t=\infty
$$

and there exists a $\Delta$-differentiable function $\eta$ such that for all constants $M>0$,

$$
\limsup _{t \rightarrow \infty} \int_{t_{0}}^{t}\left[\eta(s) p(s)(1-r(\delta(s)))^{\gamma}\left(\frac{\delta(s)}{s}\right)^{\gamma}-\frac{a(s)\left(\eta^{\Delta}(s)\right)^{2}}{4 \gamma M^{\gamma-1} \eta(s)}\right] \Delta s=\infty
$$

Then every solution of (1.10) is oscillatory.

We note that in [34], the author gave an open problem, that is, how to establish oscillation criteria for (1.10) when $\gamma<1$.

In [35], the author examined the oscillation of the second-order neutral delay dynamic equations

$$
(x(t)-r x(\tau(t)))^{\Delta \Delta}+H\left(t, x\left(h_{1}(t)\right)\right)=0, \quad t \in \mathbb{T} .
$$

The author assumes that

$\left(H_{1}\right) \tau$ and $h_{1} \in C_{\mathrm{rd}}(\mathbb{T}, \mathbb{T}), \tau(t)<t, \tau(t) \rightarrow \infty$ as $t \rightarrow \infty, h_{1}(t)<t, h_{1}(t) \rightarrow \infty$ as $t \rightarrow \infty$, and $0 \leq r<1$;

$\left(H_{2}\right) H \in C(\mathbb{T} \times \mathbb{R}, \mathbb{R})$ for each $t \in \mathbb{T}$ which are nondecreasing in $u$, and $H(t, u)>0$, for $u>0$;

$\left(H_{3}\right)|H(t, u)| \geq \alpha(t)|u|^{\lambda}$, where $\alpha(t) \geq 0$, and $0 \leq \lambda=p / q<1$ with $p, q$ being odd integers.

The main result for the oscillation of (1.13) in [35] is the following.

Theorem 1.2 (see, [35, Theorem 3.4]). Assume that $\left(H_{1}\right)-\left(H_{3}\right)$ hold. If for all sufficiently large $t_{1} \geq t_{0}$,

$$
\int_{t_{1}}^{\infty} \alpha(s)\left(\tau\left(h_{1}(s)\right)\right)^{\lambda} \Delta s=\infty
$$

then (1.13) oscillates. 
We find that the conclusion of this theorem is wrong. The following is a counter example of this theorem.

Counter Example. Consider the second-order differential equation

$$
\left(x(t)-\frac{1}{3} x\left(\frac{t}{3}\right)\right)^{\prime \prime}+\left(\frac{1}{27} e^{-1 / 3}-e^{-1 / 3} e^{-2 t / 3}\right) x^{1 / 3}(t-1)=0, \quad t \geq t_{0} .
$$

Let $\alpha(t)=e^{-1 / 3} / 27-e^{-1 / 3} e^{-2 t / 3}, r(t)=1 / 3, \tau(t)=t / 3$, and $h_{1}(t)=t-1, \lambda=1 / 3$. For all sufficiently large $t_{1} \geq t_{0}$, we find that

$$
\int_{t_{1}}^{\infty} \alpha(s)\left(\tau\left(h_{1}(s)\right)\right)^{\lambda} \Delta s=\int_{t_{1}}^{\infty} \alpha(s)\left(\tau\left(h_{1}(s)\right)\right)^{\lambda} \mathrm{d} s=\int_{t_{1}}^{\infty}\left(\frac{1}{27} e^{-1 / 3}-e^{-1 / 3} e^{-2 s / 3}\right)\left(\frac{s-1}{3}\right)^{1 / 3} \mathrm{~d} s
$$

It is easy to see that

$$
\begin{gathered}
\int_{t_{1}}^{\infty} \frac{1}{27} e^{-1 / 3}\left(\frac{s-1}{3}\right)^{1 / 3} \mathrm{~d} s=\infty \\
\int_{t_{1}}^{\infty} e^{-2 s / 3}\left(\frac{s-1}{3}\right)^{1 / 3} \mathrm{~d} s \leq \int_{t_{1}}^{\infty} e^{-2 s / 3} s^{1 / 3} \mathrm{~d} s .
\end{gathered}
$$

Integrating by parts, we obtain

$$
\int_{t_{1}}^{\infty} e^{-2 s / 3} s^{1 / 3} \mathrm{~d} s=-t_{1}^{1 / 3}\left(\frac{3}{2} e^{-2 t_{1} / 3}\right)+\frac{1}{2} \int_{t_{1}}^{\infty} e^{-2 s / 3} s^{-2 / 3} \mathrm{~d} s<\infty
$$

Hence

$$
\int_{t_{1}}^{\infty} \alpha(s)\left(\tau\left(h_{1}(s)\right)\right)^{\lambda} \mathrm{d} s=\infty
$$

Therefore, by the above theorem, (1.15) is oscillatory. However, $x(t)=\mathrm{e}^{-t}$ is a positive solution of (1.15). Therefore, the above theorem is wrong. Tracing the error to its source, we find that the following false assertion was used in the proof of the aforementioned theorem.

\section{Assertion A}

If $x$ is an eventually positive solution of (1.13), then $z(t)=x(t)-r(t) x(\tau(t))$ is eventually positive.

Abdalla [37] studied the second-order superlinear neutral delay differential equations

$$
\left[a(t)(y(t)+r(t) y(\tau(t)))^{\prime}\right]^{\prime}+p(t)|y(\delta(t))|^{\gamma} \operatorname{sign} y(\delta(t))=0, \quad t \in\left[t_{0}, \infty\right) .
$$


Most of the oscillation criteria are unsatisfactory since additional assumptions have to be imposed on the unknown solutions. Also, the author proved that if

$$
\int_{t_{0}}^{\infty} \frac{\mathrm{d} t}{a(t)}=\int_{t_{0}}^{\infty} p(t) \mathrm{d} t=\infty
$$

then every solution of (1.20) oscillates for every $r(t)>0$, but one can easily see that this result cannot be applied when $p(t)=t^{-\alpha}$ for $\alpha>1$.

Lin [38] considered the second-order nonlinear neutral differential equations

$$
[x(t)-p(t) x(t-\tau)]^{\prime \prime}+q(t) f(x(t-\sigma))=0, \quad t \geq 0,
$$

where $0 \leq p(t) \leq 1, q(t) \geq 0, \tau, \sigma>0$. The author investigated the oscillation for (1.22) when $f$ is superlinear.

Wong $[46,47]$ studied the second-order neutral differential equations

$$
[y(t)-p y(t-\tau)]^{\prime \prime}+q(t) f(y(t-\sigma))=0, \quad t \geq 0,
$$

$q \in C[0, \infty), q(t) \geq 0, f \in C^{1}(-\infty, \infty), y f(y)>0$ whenever $y \neq 0, f^{\prime}(y) \geq 0$ for all $y$, and $0<p<1, \tau>0, \sigma>0$ are constants.

The main results for the oscillation of $(1.23)$ in $[46,47]$ are the following.

Theorem 1.3 (see, [46, 47, Theorem 1]). Suppose that $f$ is superlinear. Then a solution of (1.23) is either oscillatory or tends to zero if and only if

$$
\int^{\infty} t q(t) \mathrm{d} t=\infty
$$

Theorem 1.4 (see, [46, 47, Theorem 2]). Suppose that $f$ is sublinear and in addition satisfies

$$
f(u v) \geq f(u) f(v), \quad u v \geq 0
$$

Then a solution of (1.23) is either oscillatory or tends to zero if and only if

$$
\int^{\infty} f(t) q(t) \mathrm{d} t=\infty
$$

$\mathrm{Li}$ and Saker [40] investigated the second-order sublinear neutral delay difference equations

$$
\Delta\left(a_{n} \Delta\left(x_{n}+p_{n} x_{n-\tau}\right)\right)+q_{n} x_{n-\sigma}^{\gamma}=0,
$$

where $0<\gamma<1$ is a quotient of odd positive integers, $a_{n}>0, \Delta a_{n} \geq 0, \sum_{n=0}^{\infty} 1 / a_{n}=\infty, 0 \leq$ $p_{n}<1$, for all $n \geq 0$ and $q_{n} \geq 0$.

The main result for the oscillation of (1.27) in [40] is the following. 
Theorem 1.5 (see, [40, Theorem 2.1]). Assume that there exists a positive sequence $\left\{\rho_{n}\right\}$ such that for every $\alpha \geq 1$,

$$
\limsup _{n \rightarrow \infty} \sum_{l=0}^{n}\left[\rho_{l} Q_{l}-\frac{a_{l-\sigma}(\alpha(l+1-\sigma))^{1-\gamma}\left(\Delta \rho_{l}\right)^{2}}{4 \gamma \rho_{l}}\right]=\infty,
$$

where $Q_{n}=q_{n}\left(1-p_{n-\sigma}\right)^{\gamma}$. Then every solution of (1.27) oscillates.

Yildiz and Öcalan [41] studied the higher-order sublinear neutral delay difference equations of the type

$$
\Delta^{m}\left(y_{n}+p_{n} y_{n-l}\right)+q_{n} y_{n-k}^{\alpha}=0, \quad n \in \mathbb{N}
$$

where $0<\alpha<1$ is a ratio of odd positive integers. The authors established some oscillation criteria of (1.29).

The main results for the oscillation of (1.29) when $m=2$ in [41] are the following.

Theorem 1.6 (see, [41, Theorem 2.1(a), $m=2]$ ). Assume that $0 \leq p_{n}<1$, and

$$
\sum_{n=0}^{\infty} q_{n}\left[\left(1-p_{n-k}\right) n\right]^{\alpha}=\infty
$$

Then all solutions of (1.29) are oscillatory.

Theorem 1.7 (see, [41, Theorem 2.2, $m=2]$ ). Assume that $-1<-p_{2} \leq p_{n} \leq 0$, where $p_{2}>0$ is a constant, and

$$
\sum_{n=0}^{\infty} q_{n} n^{\alpha}=\infty
$$

Then every solution of (1.29) either oscillates or tends to zero as $n \rightarrow \infty$. equations

Cheng [42] considered the oscillation of the second-order nonlinear neutral difference

$$
\Delta\left(p_{n}\left(\Delta\left(x_{n}+c_{n} x_{n-\tau}\right)\right)^{\gamma}\right)+q_{n} x_{n-\sigma}^{\beta}=0
$$

and established some oscillation criteria of (1.32) by means of Riccati transformation techniques.

Following this trend, in this paper, we are concerned with oscillation of the secondorder neutral delay dynamic equations of Emden-Fowler type

$$
\left[a(t)(x(t)+r(t) x(\tau(t)))^{\Delta}\right]^{\Delta}+p(t) x^{\gamma}(\delta(t))=0, \quad t \in \mathbb{T} .
$$


As we are interested in oscillatory behavior, we assume throughout this paper that the given time scales $\mathbb{T}$ are unbounded above; that is, it is a time scale interval of the form $\left[t_{0}, \infty\right)$ with $t_{0} \in \mathbb{T}$.

We assume that $\gamma>0$ is a quotient of odd positive integers, the delay functions $\tau$ and $\delta$ satisfy $\tau: \mathbb{T} \rightarrow \mathbb{T}, \delta: \mathbb{T} \rightarrow \mathbb{T}, \tau(t) \leq t, \delta(t) \leq t$ for all $t \in \mathbb{T}$, and $\lim _{t \rightarrow \infty} \tau(t)=$ $\lim _{t \rightarrow \infty} \delta(t)=\infty ; a, r$ and $p$ are real-valued rd-continuous functions defined on $\mathbb{T}, a(t)>$ $0, p(t)>0, \int_{t_{0}}^{\infty} \Delta t / a(t)=\infty$.

We note that if $\mathbb{T}=\mathbb{R}$, then $\sigma(t)=t, \mu(t)=0, x^{\Delta}(t)=x^{\prime}(t)$, and (1.33) becomes the second-order nonlinear delay differential equation

$$
\left[a(t)(x(t)+r(t) x(\tau(t)))^{\prime}\right]^{\prime}+p(t) x^{\gamma}(\delta(t))=0, \quad t \in \mathbb{R} .
$$

If $\mathbb{T}=\mathbb{Z}$, then $\sigma(t)=t+1, \mu(t)=1, x^{\Delta}(t)=\Delta x(t)=x(t+1)-x(t)$, and (1.33) becomes the second-order nonlinear delay differential equation

$$
\Delta[a(t) \Delta(x(t)+r(t) x(\tau(t)))]+p(t) x^{\gamma}(\delta(t))=0, \quad t \in \mathbb{Z} .
$$

In the case of $\gamma>1,(1.33)$ is the prototype of a wide class of nonlinear dynamic equations called Emden-Fowler sublinear dynamic equations, and if $\gamma<1$, (1.33) is the prototype of dynamic equations called Emden-Fowler sublinear dynamic equations. It is interesting to study (1.33) because the continuous version, that is, (1.34), has several physical applications; see, for example, [1,39], and when $t$ is a discrete variable, it is (1.35), and it is also important in applications.

\section{Main Results}

In this section, we give some new oscillation criteria of (1.33). In order to prove our main results, we will use the formula

$$
\left((x(t))^{\gamma}\right)^{\Delta}=\gamma \int_{0}^{1}\left[h x^{\sigma}(t)+(1-h) x(t)\right]^{\gamma-1} x^{\Delta}(t) \mathrm{d} h,
$$

which is a simple consequence of Keller's chain rule [3, Theorem 1.90]. Also, we need the following auxiliary results.

For the sake of convenience, we assume that

$$
z(t)=x(t)+r(t) x(\tau(t)), \quad R\left(t, t_{*}\right)=a(t) \int_{t_{*}}^{t} \frac{\Delta s}{a(s)}, \quad \alpha\left(t, t_{*}\right)=\frac{\int_{t_{*}}^{\delta(t)} \Delta s / a(s)}{\int_{t_{*}}^{t} \Delta s / a(s)}, \quad t_{*} \geq t_{0} .
$$

Lemma 2.1. Assume that (1.11) holds, $a^{\Delta}(t) \geq 0$, and $0 \leq r(t)<1$. Then an eventually positive solution $x$ of (1.33) eventually satisfies that

$$
z(t) \geq t z^{\Delta}(t)>0, \quad z^{\Delta \Delta}(t)<0, \quad\left(a(t) z^{\Delta}(t)\right)^{\Delta}<0, \quad \frac{z(t)}{t} \quad \text { is nonincreasing. }
$$


Proof. From (1.11), the proof is similar to that of Saker et al. [24, Lemma 2.1], so it is omitted.

Lemma 2.2. Assume that

$$
\int_{t_{0}}^{\infty} p(t) \delta^{\gamma}(t) \Delta t=\infty
$$

$a^{\Delta}(t) \geq 0,-1<-r_{0} \leq r(t) \leq 0$, and $\lim _{t \rightarrow \infty} r(t)=r_{1}>-1$. Then an eventually positive solution $x$ of (1.33) eventually satisfies that

$$
z(t) \geq t z^{\Delta}(t)>0, \quad z^{\Delta \Delta}(t)<0, \quad\left(a(t) z^{\Delta}(t)\right)^{\Delta}<0, \quad \frac{z(t)}{t} \quad \text { is nonincreasing }
$$

or $\lim _{t \rightarrow \infty} x(t)=0$.

Proof. Let $x$ be an eventually positive solution of (1.33). Then there exists $t_{1} \geq t_{0}$ such that $x(t)>0, x(\tau(t))>0$, and $x(\delta(t))>0$ for all $t \geq t_{1}$. Assume that $\lim _{t \rightarrow \infty} x(t) \neq 0$, that is, $\lim \sup _{t \rightarrow \infty} x(t)>0$. Then, we have to show that (2.5) holds. It follows from (1.33) that

$$
\left(a(t) z^{\Delta}(t)\right)^{\Delta}=-p(t) x^{\gamma}(\delta(t))<0, \quad t \geq t_{1}
$$

which implies that $a z^{\Delta}$ is nonincreasing on $\left[t_{1}, \infty\right)_{\mathbb{T}}$. Since the function $a$ is nondecreasing, $z^{\Delta}$ must be nonincreasing on $\left[t_{1}, \infty\right)_{\mathbb{T}}$, that is, $z^{\Delta}$ is eventually either positive or negative. In both cases, $z$ is eventually monotonic, so that $z$ has a limit at infinity (finite or infinite). This implies that $\lim _{t \rightarrow \infty} z(t) \neq 0$; that is, $z$ is eventually positive (see [19, Lemma 3]). Then we proceed as in the proof of [24, Lemma 2.1] to obtain (2.5). The proof is complete.

Lemma 2.3. Assume that $0 \leq r(t)<1$. Further, $x$ is an eventually positive solution of (1.33). Then there exists a $t_{*} \geq t_{0}$ such that for $t \geq t_{*}$

$$
z^{\Delta}(t)>0, \quad\left(a(t) z^{\Delta}(t)\right)^{\Delta}<0, \quad z(t) \geq R\left(t, t_{*}\right) z^{\Delta}(t), \quad z(\delta(t)) \geq \alpha\left(t, t_{*}\right) z(t) .
$$

Proof. Let $x$ be an eventually positive solution of (1.33). Then there exists $t_{1} \geq t_{0}$ such that $x(t)>0, x(\tau(t))>0$, and $x(\delta(t))>0$ for all $t \geq t_{1}$. It follows from (1.33) that (2.6) holds. From (2.6), we know that $a(t) z^{\Delta}(t)$ is an eventually decreasing function. We claim that $z^{\Delta}(t)>0$ eventually. Otherwise, if there exists a $t_{2} \geq t_{1}$ such that $z^{\Delta}(t)<0$, by (2.6), we have

$$
a(t) z^{\Delta}(t) \leq a\left(t_{2}\right) z^{\Delta}\left(t_{2}\right)=b<0, \quad t \geq t_{2} .
$$

Thus

$$
z^{\Delta}(t) \leq b \frac{1}{a(t)}
$$


Integrating the above inequality from $t_{2}$ to $t$ leads to $\lim _{t \rightarrow \infty} z(t)=-\infty$, which contradicts $z(t)>0$. Hence, $z^{\Delta}(t)>0$ on $\left[t_{2}, \infty\right)_{\mathbb{T}}$. Therefore,

$$
z(t)>z(t)-z\left(t_{2}\right)=\int_{t_{2}}^{t} \frac{a(s) z^{\Delta}(s)}{a(s)} \Delta s \geq\left(a(t) z^{\Delta}(t)\right) \int_{t_{2}}^{t} \frac{\Delta s}{a(s)}
$$

which yields

$$
z(t) \geq\left(a(t) \int_{t_{2}}^{t} \frac{\Delta s}{a(s)}\right) z^{\Delta}(t)
$$

Since $a(t) z^{\Delta}(t)$ is strictly decreasing, we have

$$
z(t)-z(\delta(t))=\int_{\delta(t)}^{t} \frac{a(s) z^{\Delta}(s)}{a(s)} \Delta s \leq a(\delta(t)) z^{\Delta}(\delta(t)) \int_{\delta(t)}^{t} \frac{\Delta s}{a(s)}
$$

and so

$$
\frac{z(t)}{z(\delta(t))} \leq 1+\frac{a(\delta(t)) z^{\Delta}(\delta(t))}{z(\delta(t))} \int_{\delta(t)}^{t} \frac{\Delta s}{a(s)}
$$

Also, we have that for large $t$,

$$
z(\delta(t)) \geq z(\delta(t))-z\left(t_{2}\right)=\int_{t_{2}}^{\delta(t)} \frac{a(s) z^{\Delta}(s)}{a(s)} \Delta s \geq a(\delta(t)) z^{\Delta}(\delta(t)) \int_{t_{2}}^{\delta(t)} \frac{\Delta s}{a(s)}
$$

so we obtain

$$
\frac{a(\delta(t)) z^{\Delta}(\delta(t))}{z(\delta(t))} \leq\left(\int_{t_{2}}^{\delta(t)} \frac{\Delta s}{a(s)}\right)^{-1} .
$$

Therefore, from (2.13), we have

$$
z(\delta(t)) \geq \alpha\left(t, t_{2}\right) z(t)
$$

This completes the proof.

Lemma 2.4. Assume that $-1<-r_{0} \leq r(t) \leq 0, \lim _{t \rightarrow \infty} r(t)=r_{1}>-1$. Then an eventually positive solution $x$ of (1.33) satisfies that, for sufficiently large $t_{*} \geq t_{0}$,

$$
z^{\Delta}(t)>0, \quad\left(a(t) z^{\Delta}(t)\right)^{\Delta}<0, \quad z(t) \geq R\left(t, t_{*}\right) z^{\Delta}(t), \quad z(\delta(t)) \geq \alpha\left(t, t_{*}\right) z(t), \quad t \geq t_{*}
$$

or $\lim _{t \rightarrow \infty} x(t)=0$. 
Proof. The proof is similar to that of the proof Lemmas 2.2 and 2.3, so we omit the details.

Theorem 2.5. Assume that (1.11) holds, $a^{\Delta}(t) \geq 0$, and $0 \leq r(t)<1$. Then every solution of (1.33) oscillates if the inequality

$$
y^{\Delta}(t)+A(t) y^{\gamma}(\delta(t)) \leq 0
$$

where

$$
A(t)=p(t)(1-r(\delta(t)))^{\gamma} \frac{(\delta(t))^{\gamma}}{(a(\delta(t)))^{\gamma}}
$$

has no eventually positive solution.

Proof. Suppose to the contrary that (1.33) has a nonoscillatory solution $x$. We may assume without loss of generality that there exists $t_{1} \geq t_{0}$ such that $x(t)>0, x(\tau(t))>0$ and $x(\delta(t))>$ 0 for all $t \geq t_{1}$. From Lemma 2.1, there is some $t_{2} \geq t_{1}$ such that

$$
x(t)=z(t)-r(t) x(\tau(t)) \geq z(t)-r(t) z(\tau(t)) \geq(1-r(t)) z(t), \quad t \geq t_{2} .
$$

From (1.33), there exists a $t_{3} \geq t_{2}$ such that

$$
\left(a(t) z^{\Delta}(t)\right)^{\Delta}+p(t)(1-r(\delta(t)))^{\gamma}(z(\delta(t)))^{\gamma} \leq 0, \quad t \geq t_{3} .
$$

By Lemma 2.1, there exists a $t_{4} \geq t_{3}$ such that

$$
z(\delta(t)) \geq \delta(t) z^{\Delta}(\delta(t))
$$

Substituting the last inequality in (2.21) we obtain for $t \geq t_{4}$ that

$$
\left(a(t) z^{\Delta}(t)\right)^{\Delta}+p(t)(1-r(\delta(t)))^{\gamma}(\delta(t))^{\gamma}\left(z^{\Delta}(\delta(t))\right)^{\gamma} \leq 0 .
$$

Set $y(t)=a(t) z^{\Delta}(t)$. Then from (2.23), $y$ is positive and satisfies the inequality (2.18), and this contradicts the assumption of our theorem. Thus every solution of (1.33) oscillates. This completes the proof.

By [41, Lemma 1.1] and Theorem 2.5 in this paper, we have the following result.

Corollary 2.6. If $\mathbb{T}=\mathbb{Z}, a(t)=1, \delta(t)=t-l, l$ is a positive integer, and $0 \leq r(t)<1$, then every solution of (1.33) oscillates if

$$
\sum_{t=n_{0}}^{\infty} t^{\gamma} p(t)(1-r(\delta(t)))^{\gamma}=\infty
$$


Theorem 2.7. Assume that (2.4) holds, and $a^{\Delta}(t) \geq 0,-1<-r_{0} \leq r(t) \leq 0$, and $\lim _{t \rightarrow \infty} r(t)=$ $r_{1}>-1$. Then every solution of (1.33) either oscillates or tends to zero as $t \rightarrow \infty$ if the inequality

$$
y^{\Delta}(t)+B(t) y^{\gamma}(\delta(t)) \leq 0
$$

where

$$
B(t)=p(t) \frac{(\delta(t))^{\gamma}}{(a(\delta(t)))^{\gamma}}
$$

has no eventually positive solution.

Proof. Suppose to the contrary that (1.33) has a nonoscillatory solution $x$. We may assume without loss of generality that there exists $t_{1} \geq t_{0}$ such that $x(t)>0, x(\tau(t))>0$, and $x(\delta(t))>$ 0 for all $t \geq t_{1}$.

From Lemma 2.2, if (i) holds, there is some $t_{2} \geq t_{1}$ such that

$$
x(t)=z(t)-r(t) x(\tau(t)) \geq z(t)>0, \quad t \geq t_{2} .
$$

From (1.33), there exists a $t_{3} \geq t_{2}$ such that

$$
\left(a(t) z^{\Delta}(t)\right)^{\Delta}+p(t)(z(\delta(t)))^{\gamma} \leq 0, \quad t \geq t_{3} .
$$

By Lemma 2.2, there exists a $t_{3} \geq t_{2}$ such that

$$
z(\delta(t)) \geq \delta(t) z^{\Delta}(\delta(t))
$$

Substituting the last inequality in (2.28), we obtain for $t \geq t_{3}$ that

$$
\left(a(t) z^{\Delta}(t)\right)^{\Delta}+p(t)(\delta(t))^{\gamma}\left(z^{\Delta}(\delta(t))\right)^{\gamma} \leq 0 .
$$

Set $y(t)=a(t) z^{\Delta}(t)$. Then from (2.30), $y$ is positive and satisfies the inequality (2.25), and this contradicts the assumption of our theorem.

If (ii) holds, by Lemma 2.2, we have $\lim _{t \rightarrow \infty} x(t)=0$. This completes the proof.

By [41, Lemma 1.1] and Theorem 2.7 in this paper, we have the following result.

Corollary 2.8. Assume that $\mathbb{T}=\mathbb{Z}, a(t)=1, \delta(t)=t-l, l$ is a positive integer, $-1<-r_{0} \leq r(t) \leq 0$, and $\lim _{t \rightarrow \infty} r(t)=r>-1$. Then every solution of (1.33) either oscillates or tends to zero as $t \rightarrow \infty$ if

$$
\sum_{t=n_{0}}^{\infty} t^{r} p(t)=\infty
$$

Remark 2.9. Theorems 2.5 and 2.7 reduce the question of (1.33) to the absence of eventually positive solution (the oscillatory) of the differential inequalities (2.18) and (2.25). 
Remark 2.10. From Theorem 2.5, Theorem 2.7, and the results given in $[7-9,12,14]$, we can obtain some oscillation criteria for (1.33) in the case when $\gamma=1, a^{\Delta}(t) \geq 0$.

Theorem 2.11. Assume that (1.11) holds, $\gamma<1, a^{\Delta}(t) \geq 0$, and $0 \leq r(t)<1$. Then every solution of (1.33) oscillates if

$$
\int_{t_{0}}^{\infty} \frac{p(s)}{(a(\delta(s)))^{\gamma}}(1-r(\delta(s)))^{\gamma}(\delta(s))^{\gamma} \Delta s=\infty
$$

Proof. We assume that (1.33) has a nonoscillatory solution such that $x(t)>0, x(\tau(t))>0$, and $x(\delta(t))>0$ for all $t \geq t_{1} \geq t_{0}$. By proceeding as in the proof of Theorem 2.5, we get (2.21). By Lemma 2.1, note that $\left(a(t) z^{\Delta}(t)\right)^{\Delta}<0$, and from Keller's chain rule, we obtain

$$
\begin{aligned}
\left(\left(a(t) z^{\Delta}(t)\right)^{1-\gamma}\right)^{\Delta} & =(1-\gamma) \int_{0}^{1}\left[h\left(a(t) z^{\Delta}(t)\right)^{\sigma}+(1-h) a(t) z^{\Delta}(t)\right]^{-\gamma}\left(a(t) z^{\Delta}(t)\right)^{\Delta} \mathrm{d} h \\
& \leq(1-\gamma) \int_{0}^{1}\left[h a(t) z^{\Delta}(t)+(1-h) a(t) z^{\Delta}(t)\right]^{-\gamma}\left(a(t) z^{\Delta}(t)\right)^{\Delta} \mathrm{d} h \\
& =(1-\gamma)\left(a(t) z^{\Delta}(t)\right)^{-\gamma}\left(a(t) z^{\Delta}(t)\right)^{\Delta}<0,
\end{aligned}
$$

so

$$
\left(a(t) z^{\Delta}(t)\right)^{-\gamma}\left(a(t) z^{\Delta}(t)\right)^{\Delta} \geq \frac{\left(\left(a(t) z^{\Delta}(t)\right)^{1-\gamma}\right)^{\Delta}}{1-\gamma} .
$$

Using (2.21), we have

$$
\begin{aligned}
0 & \geq \frac{\left(a(t) z^{\Delta}(t)\right)^{\Delta}+p(t)(1-r(\delta(t)))^{\gamma}(z(\delta(t)))^{\gamma}}{\left(a(t) z^{\Delta}(t)\right)^{\gamma}} \\
& =\left(a(t) z^{\Delta}(t)\right)^{-\gamma}\left(a(t) z^{\Delta}(t)\right)^{\Delta}+p(t)(1-r(\delta(t)))^{\gamma}\left(\frac{z(\delta(t))}{a(t) z^{\Delta}(t)}\right)^{\gamma} \\
& \geq \frac{\left(\left(a(t) z^{\Delta}(t)\right)^{1-\gamma}\right)^{\Delta}}{1-\gamma}+\frac{p(t)}{(a(\delta(t)))^{\gamma}}(1-r(\delta(t)))^{\gamma}(\delta(t))^{\gamma}
\end{aligned}
$$

Hence,

$$
\frac{p(t)}{(a(\delta(t)))^{\gamma}}(1-r(\delta(t)))^{\gamma}(\delta(t))^{\gamma} \leq \frac{\left(\left(a(t) z^{\Delta}(t)\right)^{1-\gamma}\right)^{\Delta}}{\gamma-1} .
$$


Upon integration we arrive at

$$
\int_{t_{1}}^{t} \frac{p(s)}{(a(\delta(s)))^{\gamma}}(1-r(\delta(s)))^{\gamma}(\delta(s))^{\gamma} \Delta s \leq \int_{t_{1}}^{t} \frac{\left(\left(a(s) z^{\Delta}(s)\right)^{1-\gamma}\right)^{\Delta}}{\gamma-1} \Delta s \leq \frac{\left(a\left(t_{1}\right) z^{\Delta}\left(t_{1}\right)\right)^{1-\gamma}}{1-\gamma} .
$$

This contradicts (2.32) and finishes the proof.

Theorem 2.12. Assume that (2.4) holds, and $\gamma<1, a^{\Delta}(t) \geq 0,-1<-r_{0} \leq r(t) \leq 0$, and $\lim _{t \rightarrow \infty} r(t)=r_{1}>-1$. Then every solution of (1.33) either oscillates or tends to zero as $t \rightarrow \infty$ if

$$
\int_{t_{0}}^{\infty} \frac{p(s)}{(a(\delta(s)))^{r}}(\delta(s))^{r} \Delta s=\infty
$$

Proof. By Lemma 2.2, the proof is similar to that of the proof of Theorem 2.11, so we omit the details.

Theorem 2.13. Assume that $\gamma<1$ and $0 \leq r(t)<1$. Then every solution of (1.33) oscillates if

$$
\int_{t_{0}}^{\infty} \frac{p(s)}{(a(\delta(s)))^{\gamma}}(1-r(\delta(s)))^{\gamma}\left(R\left(\delta(s), t_{*}\right)\right)^{r} \Delta s=\infty
$$

holds for all sufficiently large $t_{*}$.

Proof. By Lemma 2.3, the proof is similar to that of the proof Theorem 2.11, so we omit the details.

Theorem 2.14. Assume that $\gamma<1,-1<-r_{0} \leq r(t) \leq 0$, and $\lim _{t \rightarrow \infty} r(t)=r_{1}>-1$. Then every solution of (1.33) either oscillates or tends to zero as $t \rightarrow \infty$ if

$$
\int_{t_{0}}^{\infty} \frac{p(s)}{(a(\delta(s)))^{\gamma}}\left(R\left(\delta(s), t_{*}\right)\right)^{r} \Delta s=\infty
$$

holds for all sufficiently large $t_{*}$.

Proof. By using Lemma 2.4 and (2.28), the proof is similar to that of the proof of Theorem 2.11, so we omit the details.

Theorem 2.15. Assume that (1.11) holds, $\gamma \geq 1, a^{\Delta}(t) \geq 0$, and $0 \leq r(t)<1$. Then every solution of (1.33) oscillates if

$$
\limsup _{t \rightarrow \infty}\left\{\frac{t}{a(t)} \int_{t}^{\infty} p(s)(1-r(\delta(s)))^{r}\left(\frac{\delta(s)}{s}\right)^{r} \Delta s\right\}=\infty .
$$

Proof. Suppose to the contrary that (1.33) has a nonoscillatory solution $x$. We may assume without loss of generality that there exists $t_{1} \geq t_{0}$ such that $x(t)>0, x(\tau(t))>0$, and 
$x(\delta(t))>0$ for all $t \geq t_{1}$. By proceeding as in the proof of Theorem 2.5, we get (2.21). Thus from Lemma 2.1, we have for $T \geq t \geq t_{1}$,

$$
\int_{t}^{T} p(s)(1-r(\delta(s)))^{\gamma}(z(\delta(s)))^{\gamma} \Delta s \leq-\int_{t}^{T}\left(a(s) z^{\Delta}(s)\right)^{\Delta} \Delta s=a(t) z^{\Delta}(t)-a(T) z^{\Delta}(T),
$$

and hence

$$
\int_{t}^{T} p(s)(1-r(\delta(s)))^{\gamma}(z(\delta(s)))^{\gamma} \Delta s \leq a(t) z^{\Delta}(t)
$$

This and Lemma 2.1 provide, for sufficiently large $t \in \mathbb{T}$,

$$
\begin{aligned}
z(t) & \geq t z^{\Delta}(t) \geq \frac{t}{a(t)} \int_{t}^{\infty} p(s)(1-r(\delta(s)))^{\gamma}(z(\delta(s)))^{\gamma} \Delta s \\
& \geq \frac{t}{a(t)} \int_{t}^{\infty} p(s)(1-r(\delta(s)))^{\gamma}\left(\frac{\delta(s)}{s}\right)^{\gamma} z^{\gamma}(s) \Delta s \\
& \geq z^{\gamma}(t)\left\{\frac{t}{a(t)} \int_{t}^{\infty} p(s)(1-r(\delta(s)))^{\gamma}\left(\frac{\delta(s)}{s}\right)^{\gamma} \Delta s\right\} .
\end{aligned}
$$

So

$$
\left\{\frac{t}{a(t)} \int_{t}^{\infty} p(s)(1-r(\delta(s)))^{\gamma}\left(\frac{\delta(s)}{s}\right)^{\gamma} \Delta s\right\} \leq\left(\frac{1}{z(t)}\right)^{\gamma-1} .
$$

We note that $r \geq 1$ and $z^{\Delta}(t)>0$ imply

$$
\left\{\frac{t}{a(t)} \int_{t}^{\infty} p(s)(1-r(\delta(s)))^{r}\left(\frac{\delta(s)}{s}\right)^{\gamma} \Delta s\right\} \leq\left(\frac{1}{z\left(t_{1}\right)}\right)^{\gamma-1}
$$

This contradicts (2.41) and completes the proof.

Theorem 2.16. Assume that (2.4) holds, and $\gamma \geq 1, a^{\Delta}(t) \geq 0,-1<-r_{0} \leq r(t) \leq 0$, and $\lim _{t \rightarrow \infty} r(t)=r_{1}>-1$. Then every solution of (1.33) either oscillates or tends to zero as $t \rightarrow \infty$ if

$$
\limsup _{t \rightarrow \infty}\left\{\frac{t}{a(t)} \int_{t}^{\infty} p(s)\left(\frac{\delta(s)}{s}\right)^{r} \Delta s\right\}=\infty
$$

Proof. By using Lemma 2.2 and (2.28), the proof is similar to that of the proof of Theorem 2.15, so we omit the details. 
Theorem 2.17. Assume that $r \geq 1,0 \leq r(t)<1$. Then every solution of (1.33) oscillates if

$$
\limsup _{t \rightarrow \infty}\left\{\frac{R\left(t, t_{*}\right)}{a(t)} \int_{t}^{\infty} p(s)(1-r(\delta(s)))^{\gamma}\left(\alpha\left(s, t_{*}\right)\right)^{\gamma} \Delta s\right\}=\infty
$$

holds for all sufficiently large $t_{*}$.

Proof. Suppose to the contrary that (1.33) has a nonoscillatory solution $x$. We may assume without loss of generality that there exists $t_{1} \geq t_{0}$ such that $x(t)>0, x(\tau(t))>0$, and $x(\delta(t))>$ 0 for all $t \geq t_{1}$. By proceeding as in the proof of Theorem 2.5, we obtain (2.21). Thus from Lemma 2.3, we have, for $T \geq t \geq t_{1}$,

$$
\int_{t}^{T} p(s)(1-r(\delta(s)))^{\gamma}(z(\delta(s)))^{\gamma} \Delta s \leq-\int_{t}^{T}\left(a(s) z^{\Delta}(s)\right)^{\Delta} \Delta s=a(t) z^{\Delta}(t)-a(T) z^{\Delta}(T),
$$

and hence

$$
\int_{t}^{T} p(s)(1-r(\delta(s)))^{\gamma}(z(\delta(s)))^{\gamma} \Delta s \leq a(t) z^{\Delta}(t)
$$

This and Lemma 2.3 provide, for sufficiently large $t \in \mathbb{T}$,

$$
\begin{aligned}
z(t) & \geq R\left(t, t_{*}\right) z^{\Delta}(t) \geq \frac{R\left(t, t_{*}\right)}{a(t)} \int_{t}^{\infty} p(s)(1-r(\delta(s)))^{\gamma}(z(\delta(s)))^{\gamma} \Delta s \\
& \geq \frac{R\left(t, t_{*}\right)}{a(t)} \int_{t}^{\infty} p(s)(1-r(\delta(s)))^{\gamma}\left(\alpha\left(s, t_{*}\right)\right)^{\gamma} z^{\gamma}(s) \Delta s \\
& \geq z^{\gamma}(t)\left\{\frac{R\left(t, t_{*}\right)}{a(t)} \int_{t}^{\infty} p(s)(1-r(\delta(s)))^{\gamma}\left(\alpha\left(s, t_{*}\right)\right)^{\gamma} \Delta s\right\} .
\end{aligned}
$$

So

$$
\left\{\frac{R\left(t, t_{*}\right)}{a(t)} \int_{t}^{\infty} p(s)(1-r(\delta(s)))^{\gamma}\left(\alpha\left(s, t_{*}\right)\right)^{\gamma} \Delta s\right\} \leq\left(\frac{1}{z(t)}\right)^{\gamma-1}
$$

We note that $\gamma \geq 1$ and $z^{\Delta}(t)>0$ imply

$$
\left\{\frac{R\left(t, t_{*}\right)}{a(t)} \int_{t}^{\infty} p(s)(1-r(\delta(s)))^{\gamma}\left(\alpha\left(s, t_{*}\right)\right)^{\gamma} \Delta s\right\} \leq\left(\frac{1}{z\left(t_{1}\right)}\right)^{\gamma-1}
$$

This contradicts (2.48) and completes the proof. 
Theorem 2.18. Assume that (2.4) holds, and $\gamma \geq 1, a^{\Delta}(t) \geq 0,-1<-r_{0} \leq r(t) \leq 0$, and $\lim _{t \rightarrow \infty} r(t)=r>-1$. Then every solution of (1.33) either oscillates or tends to zero as $t \rightarrow \infty$ if

$$
\limsup _{t \rightarrow \infty}\left\{\frac{R\left(t, t_{*}\right)}{a(t)} \int_{t}^{\infty} p(s)\left(\alpha\left(s, t_{*}\right)\right)^{r} \Delta s\right\}=\infty
$$

holds for all sufficiently large $t_{*}$.

Proof. By using Lemma 2.4 and (2.28), the proof is similar to that of the proof of Theorem 2.17, so we omit the details.

Theorem 2.19. Assume that (1.11) holds, $\gamma>1, a^{\Delta}(t) \geq 0$, and $0 \leq r(t)<1$. Then every solution of (1.33) oscillates if

$$
\int_{t_{0}}^{\infty} \sigma(s) \frac{p(s)}{a(s)}(1-r(\delta(s)))^{\gamma}\left(\frac{\delta(s)}{\sigma(s)}\right)^{\gamma} \Delta s=\infty
$$

Proof. We assume that (1.33) has a nonoscillatory solution such that $x(t)>0, x(\tau(t))>0$, and $x(\delta(t))>0$ for all $t \geq t_{1} \geq t_{0}$. By proceeding as in the proof of Theorem 2.5, we get (2.21). Define the function

$$
\omega(t)=\frac{t a(t) z^{\Delta}(t)}{z^{\gamma}(t)}, \quad t \geq t_{1}
$$

By Lemma 2.1, $\omega(t)>0$. We calculate

$$
\omega^{\Delta}(t)=\left\{a(t) z^{\Delta}(t)+\sigma(t)\left(a(t) z^{\Delta}(t)\right)^{\Delta}\right\}\left(z^{-\gamma}(t)\right)^{\sigma}+t a(t) z^{\Delta}(t)\left(z^{-\gamma}(t)\right)^{\Delta}
$$

From (2.21), we have

$$
\omega^{\Delta}(t) \leq a(t) z^{\Delta}(t)\left(z^{-\gamma}(t)\right)^{\sigma}-\sigma(t) p(t)(1-r(\delta(t)))^{\gamma}\left(\frac{z(\delta(t))}{z(\sigma(t))}\right)^{\gamma}+t a(t) z^{\Delta}(t)\left(z^{-\gamma}(t)\right)^{\Delta},
$$

and by Lemma 2.1, we have

$$
\omega^{\Delta}(t) \leq a(t) z^{\Delta}(t)\left(z^{-\gamma}(t)\right)^{\sigma}-\sigma(t) p(t)(1-r(\delta(t)))^{\gamma}\left(\frac{\delta(t)}{\sigma(t)}\right)^{\gamma}
$$

because $\left(z^{-\gamma}(t)\right)^{\Delta} \leq 0$ due to Keller's chain rule. Since

$$
\begin{aligned}
\left((z(t))^{1-\gamma}\right)^{\Delta} & =(1-\gamma) \int_{0}^{1}\left[h z^{\sigma}(t)+(1-h) z(t)\right]^{-\gamma} z^{\Delta}(t) \mathrm{d} h \\
& \leq(1-\gamma) \int_{0}^{1}\left[h z^{\sigma}(t)+(1-h) z^{\sigma}(t)\right]^{-\gamma} z^{\Delta}(t) \mathrm{d} h=(1-\gamma)\left(z^{\sigma}(t)\right)^{-\gamma} z^{\Delta}(t)
\end{aligned}
$$


thus

$$
\omega^{\Delta}(t) \leq a(t) \frac{\left((z(t))^{1-\gamma}\right)^{\Delta}}{1-\gamma}-\sigma(t) p(t)(1-r(\delta(t)))^{\gamma}\left(\frac{\delta(t)}{\sigma(t)}\right)^{\gamma} .
$$

Upon integration we arrive at

$$
\begin{aligned}
& \int_{t_{1}}^{t} \sigma(s) \frac{p(s)}{a(s)}(1-r(\delta(s)))^{\gamma}\left(\frac{\delta(s)}{\sigma(s)}\right)^{\gamma} \Delta s \\
& \quad \leq \int_{t_{1}}^{t}\left\{\frac{\left((z(s))^{1-\gamma}\right)^{\Delta}}{1-\gamma}-\frac{\omega^{\Delta}(s)}{a(s)}\right\} \Delta s \\
& \quad=\frac{(z(t))^{1-\gamma}}{1-\gamma}-\frac{\left(z\left(t_{1}\right)\right)^{1-\gamma}}{1-\gamma}-\int_{t_{1}}^{t} \frac{\omega^{\Delta}(s)}{a(s)} \Delta s \\
& \quad=\frac{(z(t))^{1-\gamma}}{1-\gamma}-\frac{\left(z\left(t_{1}\right)\right)^{1-\gamma}}{1-\gamma}-\frac{\omega(t)}{a(t)}+\frac{\omega\left(t_{1}\right)}{a\left(t_{1}\right)}+\int_{t_{1}}^{t} \omega^{\sigma}(s)\left(\frac{1}{a(s)}\right)^{\Delta} \Delta s .
\end{aligned}
$$

Noting that $(1 / a(t))^{\Delta} \leq 0$, we have

$$
\int_{t_{1}}^{t} \sigma(s) \frac{p(s)}{a(s)}(1-r(\delta(s)))^{\gamma}\left(\frac{\delta(s)}{\sigma(s)}\right)^{\gamma} \Delta s \leq \frac{\left(z\left(t_{1}\right)\right)^{1-\gamma}}{\gamma-1}+\frac{\omega\left(t_{1}\right)}{a\left(t_{1}\right)} .
$$

This contradicts (2.55) and finishes the proof.

Theorem 2.20. Assume that (2.4) holds, and $\gamma>1, a^{\Delta}(t) \geq 0,-1<-r_{0} \leq r(t) \leq 0$, and $\lim _{t \rightarrow \infty} r(t)=r_{1}>-1$. Then every solution of (1.33) either oscillates or tends to zero as $t \rightarrow \infty$ if

$$
\int_{t_{0}}^{\infty} \sigma(s) p(s)\left(\frac{\delta(s)}{\sigma(s)}\right)^{\gamma} \Delta s=\infty
$$

Proof. By using Lemma 2.2 and (2.28), the proof is similar to that of the proof of Theorem 2.19, so we omit the details.

In the following, we use a Riccati transformation technique to establish new oscillation criteria for (1.33).

Theorem 2.21. Assume that $\gamma \geq 1$, and $0 \leq r(t)<1$. Furthermore, suppose that there exists a positive $\Delta$-differentiable function $\eta$ such that for all sufficiently large $t_{*}$, and for all constants $M>0$, 
for $t_{1} \geq t_{*}$

$$
\limsup _{t \rightarrow \infty} \int_{t_{1}}^{t}\left[\eta(s) p(s)(1-r(\delta(s)))^{\gamma}\left(\alpha\left(s, t_{*}\right)\right)^{\gamma}-\frac{a(s)\left(\eta^{\Delta}(s)\right)^{2}}{4 \gamma M^{\gamma-1} \eta(s)}\right] \Delta s=\infty .
$$

Then every solution of (1.33) oscillates.

Proof. We assume that (1.33) has a nonoscillatory solution such that $x(t)>0, x(\tau(t))>0$, and $x(\delta(t))>0$ for all $t \geq t_{1} \geq t_{0}$. By proceeding as in the proof of Theorem 2.5, we get (2.21). Define the function $\omega$ by the Riccati substitution

$$
\omega(t)=\eta(t) \frac{a(t) z^{\Delta}(t)}{z^{\gamma}(t)}, \quad t \geq t_{1} .
$$

Then $\omega(t)>0$. By the product rule and then the quotient rule

$$
\begin{aligned}
\omega^{\Delta}(t) & =\left(a(t) z^{\Delta}(t)\right)^{\sigma}\left[\frac{\eta(t)}{z^{\gamma}(t)}\right]^{\Delta}+\frac{\eta(t)}{z^{\gamma}(t)}\left(a(t) z^{\Delta}(t)\right)^{\Delta} \\
& =\frac{\eta(t)}{z^{\gamma}(t)}\left(a(t) z^{\Delta}(t)\right)^{\Delta}+\left(a(t) z^{\Delta}(t)\right)^{\sigma}\left[\frac{z^{\gamma}(t) \eta^{\Delta}(t)-\eta(t)\left(z^{\gamma}(t)\right)^{\Delta}}{z^{\gamma}(t)\left(z^{\sigma}(t)\right)^{\gamma}}\right] .
\end{aligned}
$$

In view of (2.21) and (2.66), we have

$$
\omega^{\Delta}(t) \leq-\eta(t) p(t)(1-r(\delta(t)))^{\gamma}\left(\frac{z(\delta(t))}{z(t)}\right)^{\gamma}+\frac{\eta^{\Delta}(t)}{\eta^{\sigma}(t)} \omega^{\sigma}(t)-\frac{\eta(t)\left(a(t) z^{\Delta}(t)\right)^{\sigma}\left(z^{\gamma}(t)\right)^{\Delta}}{z^{\gamma}(t)\left(z^{\sigma}(t)\right)^{\gamma}}
$$

By the chain rule and $\gamma \geq 1$, we obtain

$$
\left(z^{\gamma}(t)\right)^{\Delta} \geq \gamma z^{\gamma-1}(t) z^{\Delta}(t) \geq \gamma M^{\gamma-1} z^{\Delta}(t)
$$

where $M=z\left(t_{1}\right)>0$. In view of $\left(a(t) z^{\Delta}(t)\right)^{\Delta}<0$, we have

$$
a(t) z^{\Delta}(t) \geq\left(a(t) z^{\Delta}(t)\right)^{\sigma}
$$

and by Lemma 2.3 , we see that

$$
\omega^{\Delta}(t) \leq-\eta(t) p(t)(1-r(\delta(t)))^{\gamma}\left(\alpha\left(t, t_{*}\right)\right)^{\gamma}+\frac{\eta^{\Delta}(t)}{\eta^{\sigma}(t)} \omega^{\sigma}(t)-\frac{\gamma M^{\gamma-1} \eta(t)}{a(t)\left(\eta^{\sigma}(t)\right)^{2}}\left(\omega^{\sigma}(t)\right)^{2} .
$$


Integrating (2.71) from $t_{1}$ to $t$, we obtain

$$
\begin{aligned}
\int_{t_{1}}^{t} \eta(s) p(s)(1-r(\delta(s)))^{\gamma}\left(\alpha\left(s, t_{*}\right)\right)^{r} \Delta s \\
\leq-\int_{t_{1}}^{t} \omega^{\Delta}(s) \Delta s \\
\quad+\int_{t_{1}}^{t} \frac{\eta^{\Delta}(s)}{\eta^{\sigma}(s)} \omega^{\sigma}(s) \Delta s-\int_{t_{1}}^{t} \frac{\gamma M^{\gamma-1} \eta(s)}{a(s)\left(\eta^{\sigma}(s)\right)^{2}}\left(\omega^{\sigma}(s)\right)^{2} \Delta s .
\end{aligned}
$$

Hence

$$
\int_{t_{1}}^{t}\left[\eta(s) p(s)(1-r(\delta(s)))^{\gamma}\left(\alpha\left(s, t_{*}\right)\right)^{\gamma}-\frac{a(s)\left(\eta^{\Delta}(s)\right)^{2}}{4 \gamma M^{\gamma-1} \eta(s)}\right] \Delta s \leq \omega\left(t_{1}\right),
$$

which contradicts condition (2.65). The proof is complete.

Theorem 2.22. Assume that $r \geq 1,-1<-r_{0} \leq r(t) \leq 0$, and $\lim _{t \rightarrow \infty} r(t)=r_{1}>-1$. If there exists a positive $\Delta$-differentiable function $\eta$ such that for all sufficiently large $t_{*}$, and for all constants $M>0$, for $t_{1} \geq t_{*}$

$$
\limsup _{t \rightarrow \infty} \int_{t_{1}}^{t}\left[\eta(s) p(s)\left(\alpha\left(s, t_{*}\right)\right)^{\gamma}-\frac{a(s)\left(\eta^{\Delta}(s)\right)^{2}}{4 \gamma M^{\gamma-1} \eta(s)}\right] \Delta s=\infty,
$$

then every solution of (1.33) either oscillates or tends to zero as $t \rightarrow \infty$.

Proof. By Lemma 2.4 and (2.28), the proof is similar to that of the proof of Theorem 2.21, so we omit the details.

Theorem 2.23. Assume that (1.11) holds, $\gamma \leq 1, a^{\Delta}(t) \geq 0$, and $0 \leq r(t)<1$. Furthermore, suppose that there exists a positive $\Delta$-differentiable function $\eta$ such that for all sufficiently large $t_{1}$, and for all constants $M>0$,

$$
\limsup _{t \rightarrow \infty} \int_{t_{1}}^{t}\left[\eta(s) p(s)(1-r(\delta(s)))^{\gamma}\left(\frac{\delta(s)}{s}\right)^{\gamma}-\frac{a(s)\left(\eta^{\Delta}(s)\right)^{2}}{4 \gamma M^{\gamma-1}(\sigma(s))^{\gamma-1} \eta(s)}\right] \Delta s=\infty .
$$

Then every solution of (1.33) oscillates.

Proof. We assume that (1.33) has a nonoscillatory solution such that $x(t)>0, x(\tau(t))>0$, and $x(\delta(t))>0$ for all $t \geq t_{1} \geq t_{0}$. By proceeding as in the proof of Theorem 2.5, we obtain (2.21). 
Define the function $\omega$ by the Riccati substitution as (2.66). Then $\omega(t)>0$. By the product rule and then the quotient rule

$$
\begin{aligned}
\omega^{\Delta}(t) & =\left(a(t) z^{\Delta}(t)\right)^{\sigma}\left[\frac{\eta(t)}{z^{\gamma}(t)}\right]^{\Delta}+\frac{\eta(t)}{z^{\gamma}(t)}\left(a(t) z^{\Delta}(t)\right)^{\Delta} \\
& =\frac{\eta(t)}{z^{\gamma}(t)}\left(a(t) z^{\Delta}(t)\right)^{\Delta}+\left(a(t) z^{\Delta}(t)\right)^{\sigma}\left[\frac{z^{\gamma}(t) \eta^{\Delta}(t)-\eta(t)\left(z^{\gamma}(t)\right)^{\Delta}}{z^{\gamma}(t)\left(z^{\sigma}(t)\right)^{\gamma}}\right] .
\end{aligned}
$$

In view of (2.21) and (2.66), we have

$$
\omega^{\Delta}(t) \leq-\eta(t) p(t)(1-r(\delta(t)))^{\gamma}\left(\frac{z(\delta(t))}{z(t)}\right)^{\gamma}+\frac{\eta^{\Delta}(t)}{\eta^{\sigma}(t)} \omega^{\sigma}(t)-\frac{\eta(t)\left(a(t) z^{\Delta}(t)\right)^{\sigma}\left(z^{\gamma}(t)\right)^{\Delta}}{z^{\gamma}(t)\left(z^{\sigma}(t)\right)^{\gamma}} .
$$

From the chain rule and $\gamma \leq 1$, we get

$$
\left(z^{\gamma}(t)\right)^{\Delta} \geq \gamma z^{\gamma-1}(\sigma(t)) z^{\Delta}(t)
$$

Noting that $z(t) / t$ is nonincreasing, and there exists a constant $M>0$, such that $z(t) \leq M t$, hence we have

$$
\left(z^{\gamma}(t)\right)^{\Delta} \geq \gamma z^{\gamma-1}(\sigma(t)) z^{\Delta}(t) \geq \gamma M^{\gamma-1}(\sigma(t))^{\gamma-1} z^{\Delta}(t)
$$

In view of $\left(a(t) z^{\Delta}(t)\right)^{\Delta}<0$, we have

$$
a(t) z^{\Delta}(t) \geq\left(a(t) z^{\Delta}(t)\right)^{\sigma}
$$

and by Lemma 2.1, we see that

$$
\omega^{\Delta}(t) \leq-\eta(t) p(t)(1-r(\delta(t)))^{\gamma}\left(\frac{\delta(t)}{t}\right)^{\gamma}+\frac{\eta^{\Delta}(t)}{\eta^{\sigma}(t)} \omega^{\sigma}(t)-\frac{\gamma M^{\gamma-1}(\sigma(t))^{\gamma-1} \eta(t)}{a(t)\left(\eta^{\sigma}(t)\right)^{2}}\left(\omega^{\sigma}(t)\right)^{2} .
$$

Integrating (2.81) from $t_{1}$ to $t$, we obtain

$$
\begin{aligned}
& \int_{t_{1}}^{t} \eta(s) p(s)(1-r(\delta(s)))^{\gamma}\left(\frac{\delta(s)}{s}\right)^{\gamma} \Delta s \\
& \quad \leq-\int_{t_{1}}^{t} \omega^{\Delta}(s) \Delta s+\int_{t_{1}}^{t} \frac{\eta^{\Delta}(s)}{\eta^{\sigma}(s)} \omega^{\sigma}(s) \Delta s-\int_{t_{1}}^{t} \frac{\gamma M^{\gamma-1}(\sigma(s))^{\gamma-1} \eta(s)}{a(s)\left(\eta^{\sigma}(s)\right)^{2}}\left(\omega^{\sigma}(s)\right)^{2} \Delta s .
\end{aligned}
$$


Hence

$$
\int_{t_{1}}^{t}\left[\eta(s) p(s)(1-r(\delta(s)))^{\gamma}\left(\frac{\delta(s)}{s}\right)^{\gamma}-\frac{a(s)\left(\eta^{\Delta}(s)\right)^{2}}{4 \gamma M^{\gamma-1}(\sigma(s))^{\gamma-1} \eta(s)}\right] \Delta s \leq \omega\left(t_{1}\right),
$$

which contradicts condition (2.75). The proof is complete.

Theorem 2.24. Assume that (2.4) holds, $\gamma \leq 1, a^{\Delta}(t) \geq 0,-1<-r_{0} \leq r(t) \leq$ 0 , and $\lim _{t \rightarrow \infty} r(t)=r_{1}>-1$. If there exists a positive $\Delta$-differentiable function $\eta$ such that for all sufficiently large $t_{1}$, and for all constants $M>0$,

$$
\limsup _{t \rightarrow \infty} \int_{t_{1}}^{t}\left[\eta(s) p(s)\left(\frac{\delta(s)}{s}\right)^{\gamma}-\frac{a(s)\left(\eta^{\Delta}(s)\right)^{2}}{4 \gamma M^{\gamma-1}(\sigma(s))^{\gamma-1} \eta(s)}\right] \Delta s=\infty
$$

then every solution of (1.33) either oscillates or tends to zero as $t \rightarrow \infty$.

Proof. By Lemma 2.2 and (2.28), the proof is similar to that of the proof of Theorem 2.23, so we omit the details.

Theorem 2.25. Assume that $\gamma \leq 1, a^{\Delta}(t) \leq 0$, and $0 \leq r(t)<1$. Furthermore, suppose that there exists a positive $\Delta$-differentiable function $\eta$ such that for all sufficiently large $t_{*}$, and for all constants $M>0$, for $t_{1} \geq t_{*}$,

$$
\limsup _{t \rightarrow \infty} \int_{t_{1}}^{t}\left[\eta(s) p(s)(1-r(\delta(s)))^{\gamma}\left(\alpha\left(s, t_{*}\right)\right)^{\gamma}-\frac{a(s)(\sigma(s))^{1-\gamma}\left(\eta^{\Delta}(s)\right)^{2}}{4 \gamma M^{\gamma-1}(a(\sigma(s)))^{1-\gamma} \eta(s)}\right] \Delta s=\infty .
$$

Then every solution of (1.33) oscillates.

Proof. We assume that (1.33) has a nonoscillatory solution such that $x(t)>0, x(\tau(t))>0$, and $x(\delta(t))>0$ for all $t \geq t_{1} \geq t_{0}$. By proceeding as in the proof of Theorem 2.5, we have (2.21). Define the function $\omega$ by the Riccati substitution as (2.66). Then $\omega(t)>0$. By the product rule and then the quotient rule

$$
\begin{aligned}
\omega^{\Delta}(t) & =\left(a(t) z^{\Delta}(t)\right)^{\sigma}\left[\frac{\eta(t)}{z^{\gamma}(t)}\right]^{\Delta}+\frac{\eta(t)}{z^{\gamma}(t)}\left(a(t) z^{\Delta}(t)\right)^{\Delta} \\
& =\frac{\eta(t)}{z^{\gamma}(t)}\left(a(t) z^{\Delta}(t)\right)^{\Delta}+\left(a(t) z^{\Delta}(t)\right)^{\sigma}\left[\frac{z^{\gamma}(t) \eta^{\Delta}(t)-\eta(t)\left(z^{\gamma}(t)\right)^{\Delta}}{z^{\gamma}(t)\left(z^{\sigma}(t)\right)^{\gamma}}\right] .
\end{aligned}
$$

In view of (2.21) and (2.66), we have

$$
\omega^{\Delta}(t) \leq-\eta(t) p(t)(1-r(\delta(t)))^{\gamma}\left(\frac{z(\delta(t))}{z(t)}\right)^{\gamma}+\frac{\eta^{\Delta}(t)}{\eta^{\sigma}(t)} \omega^{\sigma}(t)-\frac{\eta(t)\left(a(t) z^{\Delta}(t)\right)^{\sigma}\left(z^{\gamma}(t)\right)^{\Delta}}{z^{\gamma}(t)\left(z^{\sigma}(t)\right)^{\gamma}} .
$$


By the chain rule and $\gamma \leq 1$, we obtain

$$
\left(z^{\gamma}(t)\right)^{\Delta} \geq \gamma z^{\gamma-1}(\sigma(t)) z^{\Delta}(t)
$$

and noting that $\left(a(t) z^{\Delta}(t)\right)^{\Delta}<0$ and there exists a constant $L>0$ such that $a(t) z^{\Delta}(t) \leq L$, so

$$
z(t)=z\left(t_{1}\right)+\int_{t_{1}}^{t} z^{\Delta}(s) \Delta s \leq z\left(t_{1}\right)+\int_{t_{1}}^{t} \frac{L}{a(s)} \Delta s .
$$

From $a^{\Delta}(t) \leq 0$, there exists a positive constant $M$ such that

$$
z(t) \leq z\left(t_{1}\right)+\frac{L}{a(t)}\left(t-t_{1}\right)=\frac{z\left(t_{1}\right) a(t)+L\left(t-t_{1}\right)}{a(t)} \leq \frac{M t}{a(t)} .
$$

Hence

$$
\left(z^{\gamma}(t)\right)^{\Delta} \geq \gamma z^{\gamma-1}(\sigma(t)) z^{\Delta}(t) \geq \gamma M^{\gamma-1}\left(\frac{\sigma(t)}{a(\sigma(t))}\right)^{\gamma-1} z^{\Delta}(t) .
$$

In view of $\left(a(t) z^{\Delta}(t)\right)^{\Delta}<0$, we have

$$
a(t) z^{\Delta}(t) \geq\left(a(t) z^{\Delta}(t)\right)^{\sigma}
$$

and by Lemma 2.3, we see that

$$
\begin{aligned}
\omega^{\Delta}(t) \leq & -\eta(t) p(t)(1-r(\delta(t)))^{\gamma}\left(\alpha\left(t, t_{*}\right)\right)^{\gamma} \\
& +\frac{\eta^{\Delta}(t)}{\eta^{\sigma}(t)} \omega^{\sigma}(t)-\frac{\gamma M^{\gamma-1} \eta(t)}{a(t)\left(\eta^{\sigma}(t)\right)^{2}}\left(\frac{\sigma(t)}{a(\sigma(t))}\right)^{\gamma-1}\left(\omega^{\sigma}(t)\right)^{2} .
\end{aligned}
$$

Integrating (2.93) from $t_{1}$ to $t$, we obtain

$$
\begin{aligned}
& \int_{t_{1}}^{t} \eta(s) p(s)(1-r(\delta(s)))^{\gamma}\left(\alpha\left(s, t_{*}\right)\right)^{\gamma} \Delta s \\
& \quad \leq-\int_{t_{1}}^{t} \omega^{\Delta}(s) \Delta s+\int_{t_{1}}^{t} \frac{\eta^{\Delta}(s)}{\eta^{\sigma}(s)} \omega^{\sigma}(s) \Delta s-\int_{t_{1}}^{t} \frac{\gamma M^{\gamma-1} \eta(s)}{a(s)\left(\eta^{\sigma}(s)\right)^{2}}\left(\frac{\sigma(s)}{a(\sigma(s))}\right)^{\gamma-1}\left(\omega^{\sigma}(s)\right)^{2} \Delta s .
\end{aligned}
$$

Thus

$$
\int_{t_{1}}^{t}\left[\eta(s) p(s)(1-r(\delta(s)))^{\gamma}\left(\alpha\left(s, t_{*}\right)\right)^{\gamma}-\frac{a(s)(\sigma(s))^{1-\gamma}\left(\eta^{\Delta}(s)\right)^{2}}{4 \gamma M^{\gamma-1}(a(\sigma(s)))^{1-\gamma} \eta(s)}\right] \Delta s \leq \omega\left(t_{1}\right),
$$

which contradicts condition (2.85). The proof is complete. 
Theorem 2.26. Assume that $r \leq 1, a^{\Delta}(t) \leq 0,-1<-r_{0} \leq r(t) \leq 0$, and $\lim _{t \rightarrow \infty} r(t)=r>-1$. If there exists a positive $\Delta$-differentiable function $\eta$ such that for all sufficiently large $t_{*}$, and for all constants $M>0$, for $t_{1} \geq t_{*}$,

$$
\limsup _{t \rightarrow \infty} \int_{t_{1}}^{t}\left[\eta(s) p(s)\left(\alpha\left(s, t_{*}\right)\right)^{\gamma}-\frac{a(s)(\sigma(s))^{1-\gamma}\left(\eta^{\Delta}(s)\right)^{2}}{4 \gamma M^{-1}(a(\sigma(s)))^{1-\gamma} \eta(s)}\right] \Delta s=\infty,
$$

then every solution of (1.33) either oscillates or tends to zero as $t \rightarrow \infty$.

Proof. By Lemma 2.4 and (2.28), the proof is similar to that of the proof of Theorem 2.25, so we omit the details.

\section{Conclusions}

In this paper, we consider the oscillation of second-order Emden-Fowler neutral delay dynamic equations (1.33). In some sense, our results extend and improve the results in $[7,32,34,35,40,41]$. For example, Theorems $2.5,2.11,2.13$, and 2.23 give some answers for the open problem posed by [34] since these results can be applied to (1.33) when $\gamma<1$, Theorems $2.7,2.12,2.14,2.16,2.18,2.20,2.22,2.24$, and 2.26 correct an error in [35]. Theorem 2.15 includes the results of [7, Theorem 4.4], [32, Theorem 3.1], Theorem 2.11 includes the result of [32, Theorem 3.5], Theorem 2.11 and Corollary 2.6 include the result of [41, Theorem 2.1(a), $m=2$ ], Corollary 2.8 includes result of [41, Theorem 2.2, $m=2]$, Theorem 2.13 does not require the conditions $a^{\Delta}(t) \geq 0$, so it improves the results of [40], and Theorems 2.17 and 2.21 improve the results in [34] since these results can be applied when $a^{\Delta}(t) \leq 0$.

The main results in this paper require that $\int_{t_{0}}^{\infty} \Delta t / a(t)=\infty$; it would be interesting to find another method to study (1.33) when $\int_{t_{0}}^{\infty} \Delta t / a(t)<\infty$. Additional examples may also be given; due to the limited space, we leave this to the interested reader.

\section{Acknowledgments}

The authors sincerely thank the reviewers for their valuable suggestions and useful comments that have lead to the present improved version of the original manuscript. This research is supported by the Natural Science Foundation of China (11071143, 60904024), China Postdoctoral Science Foundation funded project (20080441126, 200902564), Shandong

Postdoctoral funded project (200802018) and supported by the Natural Science Foundation of Shandong (Y2008A28, ZR2009AL003), and by University of Jinan Research Funds for Doctors (XBS0843).

\section{References}

[1] S. Hilger, "Analysis on measure chains-a unified approach to continuous and discrete calculus," Results in Mathematics, vol. 18, no. 1-2, pp. 18-56, 1990.

[2] V. Kac and P. Cheung, Quantum Calculus, Universitext, Springer, New York, NY, USA, 2002.

[3] M. Bohner and A. Peterson, Dynamic Equations on Time Scales, Birkhäauser, Boston, Mass, USA, 2001.

[4] V. Spedding, Taming Nature's Numbers, New Scientist, 2003.

[5] R. P. Agarwal, M. Bohner, D. O’Regan, and A. Peterson, "Dynamic equations on time scales: a survey," Journal of Computational and Applied Mathematics, vol. 141, no. 1-2, pp. 1-26, 2002. 
[6] M. Bohner and A. Peterson, Advances in Dynamic Equations on Time Scales, Birkhäauser, Boston, Mass, USA, 2003.

[7] R. P. Agarwal, M. Bohner, and S. H. Saker, "Oscillation of second order delay dynamic equations," The Canadian Applied Mathematics Quarterly, vol. 13, no. 1, pp. 1-17, 2005.

[8] R. P. Agarwal and M. Bohner, "An oscillation criterion for first order delay dynamic equations," Functional Differential Equations, vol. 16, no. 1, pp. 11-17, 2009.

[9] M. Bohner, B. Karpuz, and Ö. Öcalan, "Iterated oscillation criteria for delay dynamic equations of first order," Advances in Difference Equations, vol. 2008, Article ID 458687, 12 pages, 2008.

[10] M. Bohner and S. H. Saker, "Oscillation of second order nonlinear dynamic equations on time scales," The Rocky Mountain Journal of Mathematics, vol. 34, no. 4, pp. 1239-1254, 2004.

[11] Y. Şahiner, "Oscillation of second-order delay differential equations on time scales," Nonlinear Analysis, vol. 63, no. 5-7, pp. e1073-e1080, 2005.

[12] Y. Şahiner and I. P. Stavroulakis, "Oscillations of first order delay dynamic equations," Dynamic Systems and Applications, vol. 15, no. 3-4, pp. 645-656, 2006.

[13] B. G. Zhang and S. Zhu, "Oscillation of second-order nonlinear delay dynamic equations on time scales," Computers \& Mathematics with Applications, vol. 49, no. 4, pp. 599-609, 2005.

[14] B. G. Zhang and X. Deng, "Oscillation of delay differential equations on time scales," Mathematical and Computer Modelling, vol. 36, no. 11-13, pp. 1307-1318, 2002.

[15] L. Erbe, A. Peterson, and S. H. Saker, "Oscillation criteria for second-order nonlinear dynamic equations on time scales," Journal of the London Mathematical Society, vol. 67, no. 3, pp. 701-714, 2003.

[16] L. Erbe, A. Peterson, and S. H. Saker, "Oscillation criteria for second-order nonlinear delay dynamic equations," Journal of Mathematical Analysis and Applications, vol. 333, no. 1, pp. 505-522, 2007.

[17] T. S. Hassan, "Oscillation criteria for half-linear dynamic equations on time scales," Journal of Mathematical Analysis and Applications, vol. 345, no. 1, pp. 176-185, 2008.

[18] Z. Han, T. Li, S. Sun, and F. Cao, "Oscillation criteria for third order nonlinear delay dynamic equations on time scales," Annales Polonici Mathematici, vol. 99, pp. 143-156, 2010.

[19] B. Karpuz, Ö. Öcalan, and S. Öztürk, "Comparison theorems on the oscillation and asymptotic behaviour of higher-order neutral differential equations," Glasgow Mathematical Journal, vol. 52, no. 1, pp. 107-114, 2010.

[20] R. M. Mathsen, Q.-R. Wang, and H.-W. Wu, “Oscillation for neutral dynamic functional equations on time scales," Journal of Difference Equations and Applications, vol. 10, no. 7, pp. 651-659, 2004.

[21] Z.-Q. Zhu and Q.-R. Wang, "Existence of nonoscillatory solutions to neutral dynamic equations on time scales," Journal of Mathematical Analysis and Applications, vol. 335, no. 2, pp. 751-762, 2007.

[22] R. P. Agarwal, D. O’Regan, and S. H. Saker, "Oscillation criteria for second-order nonlinear neutral delay dynamic equations," Journal of Mathematical Analysis and Applications, vol. 300, no. 1, pp. 203 217, 2004.

[23] Y. Şahíner, "Oscillation of second-order neutral delay and mixed-type dynamic equations on time scales," Advances in Difference Equations, vol. 2006, Article ID 65626, 9 pages, 2006.

[24] S. H. Saker, R. P. Agarwal, and D. O’Regan, “Oscillation results for second-order nonlinear neutral delay dynamic equations on time scales," Applicable Analysis, vol. 86, no. 1, pp. 1-17, 2007.

[25] S. H. Saker, "Oscillation of second-order nonlinear neutral delay dynamic equations on time scales," Journal of Computational and Applied Mathematics, vol. 187, no. 2, pp. 123-141, 2006.

[26] S. H. Saker and D. O’Regan, "New oscillation criteria for second-order neutral functional dynamic equations via the generalized Riccati substitution," Communications in Nonlinear Science and Numerical Simulation, vol. 16, no. 1, pp. 423-434, 2010.

[27] H.-W. Wu, R.-K. Zhuang, and R. M. Mathsen, “Oscillation criteria for second-order nonlinear neutral variable delay dynamic equations," Applied Mathematics and Computation, vol. 178, no. 2, pp. 321-331, 2006.

[28] S.-Y. Zhang and Q.-R. Wang, "Oscillation of second-order nonlinear neutral dynamic equations on time scales," Applied Mathematics and Computation, vol. 216, no. 10, pp. 2837-2848, 2010.

[29] E. Akin-Bohner and J. Hoffacker, "Oscillation properties of an Emden-Fowler type equation on discrete time scales," Journal of Difference Equations and Applications, vol. 9, no. 6, pp. 603-612, 2003.

[30] E. Akin-Bohner, M. Bohner, and S. H. Saker, "Oscillation criteria for a certain class of second order Emden-Fowler dynamic equations," Electronic Transactions on Numerical Analysis, vol. 27, pp. 1-12, 2007.

[31] S. R. Grace, R. P. Agarwal, M. Bohner, and D. O’Regan, “Oscillation of second-order strongly superlinear and strongly sublinear dynamic equations," Communications in Nonlinear Science and Numerical Simulation, vol. 14, no. 8, pp. 3463-3471, 2009. 
[32] Z. Han, S. Sun, and B. Shi, "Oscillation criteria for a class of second-order Emden-Fowler delay dynamic equations on time scales," Journal of Mathematical Analysis and Applications, vol. 334, no. 2, pp. 847-858, 2007.

[33] S. Sun, Z. Han, P. Zhao, and C. Zhang, "Oscillation for a class of second-order Emden-Fowler delay dynamic equations on time scales," Advances in Difference Equations, vol. 2010, Article ID 642356, 15 pages, 2010.

[34] S. H. Saker, "Oscillation of second-order neutral delay dynamic equations of Emden-Fowler type," Dynamic Systems and Applications, vol. 15, no. 3-4, pp. 629-644, 2006.

[35] H. A. Agwo, "Oscillation of nonlinear second order neutral delay dynamic equations on time scales," Bulletin of the Korean Mathematical Society, vol. 45, no. 2, pp. 299-312, 2008.

[36] Z. Han, T. Li, S. Sun, and C. Zhang, "Oscillation behavior of third-order neutral Emden-Fowler delay dynamic equations on time scales," Advances in Difference Equations, vol. 2010, Article ID 586312, 23 pages, 2010.

[37] S. H. Abdallah, "Oscillatory and non-oscillatory behaviour of second-order neutral delay differential equations," Applied Mathematics and Computation, vol. 135, no. 2-3, pp. 333-344, 2003.

[38] X. Lin, "Oscillation of second-order nonlinear neutral differential equations," Journal of Mathematical Analysis and Applications, vol. 309, no. 2, pp. 442-452, 2005.

[39] J. S. W. Wong, "On the generalized Emden-Fowler equation," SIAM Review, vol. 17, pp. 339-360, 1975.

[40] W.-T. Li and S. H. Saker, "Oscillation of second-order sublinear neutral delay difference equations," Applied Mathematics and Computation, vol. 146, no. 2-3, pp. 543-551, 2003.

[41] M. K. Yildiz and Ö. Öcalan, "Oscillation results for higher order nonlinear neutral delay difference equations," Applied Mathematics Letters, vol. 20, no. 3, pp. 243-247, 2007.

[42] J. Cheng, "Kamenev-type oscillation criteria for delay difference equations," Acta Mathematica Scientia, vol. 27, no. 3, pp. 574-580, 2007.

[43] H.-F. Huo and W.-T. Li, “Oscillation of the Emden-Fowler difference systems," Journal of Mathematical Analysis and Applications, vol. 256, no. 2, pp. 478-485, 2001.

[44] W.-T. Li and S. S. Cheng, "Asymptotically linear solutions of a discrete Emden-Fowler equation," Far East Journal of Mathematical Sciences, vol. 6, no. 4, pp. 521-542, 1998.

[45] S. H. Saker, "Kamenev-type oscillation criteria for forced Emden-Fowler superlinear difference equations," Electronic Journal of Differential Equations, vol. 2006, no. 68, pp. 1-9, 2002.

[46] J. S. W. Wong, "Necessary and sufficient conditions for oscillation of second order neutral differential equations [Journal of Mathematical Analysis and Applications, vol. 252, no.1, pp. 342-352, 2002]," Journal of Mathematical Analysis and Applications, vol. 252, no. 1, pp. 342-352, 2002.

[47] J. S. W. Wong, "Corrigendum to: "Necessary and sufficient conditions for oscillation of second order neutral differential equations"," Journal of Mathematical Analysis and Applications, vol. 332, no. 1, pp. 751-752, 2007. 


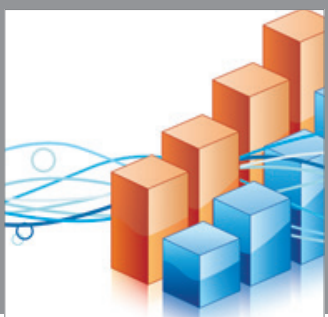

Advances in

Operations Research

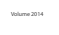

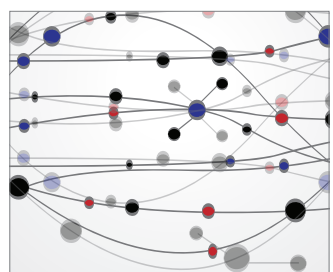

\section{The Scientific} World Journal
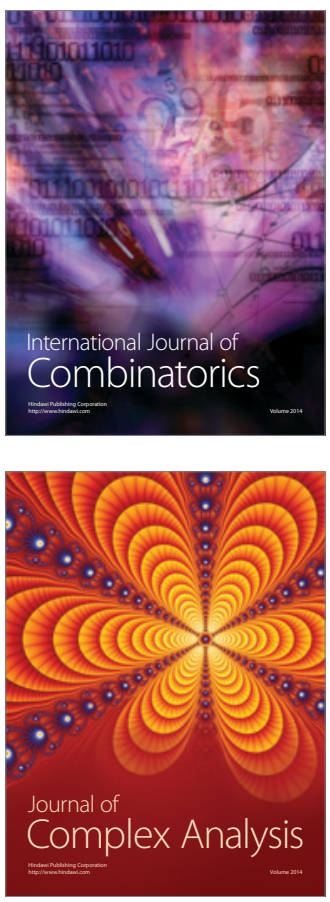

International Journal of

Mathematics and

Mathematical

Sciences
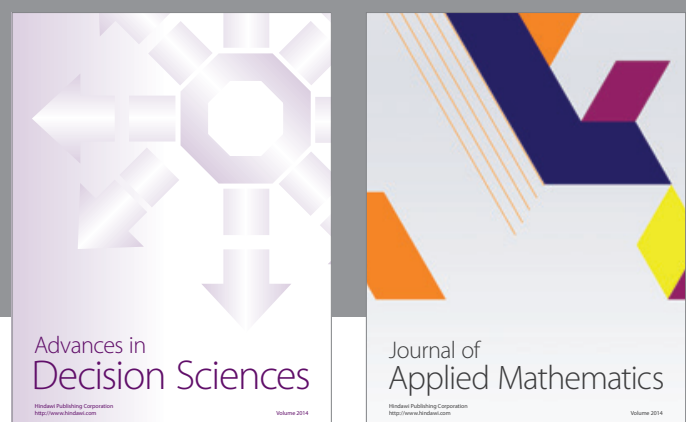

Journal of

Applied Mathematics
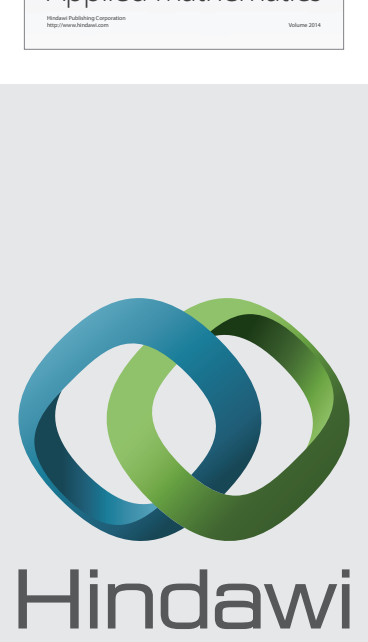

Submit your manuscripts at http://www.hindawi.com
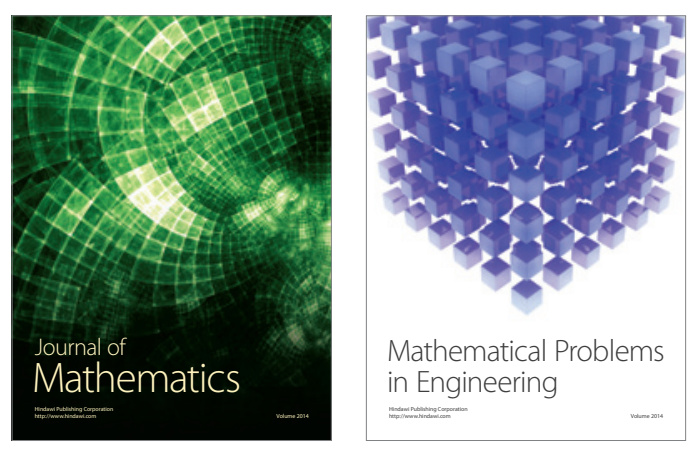

Mathematical Problems in Engineering
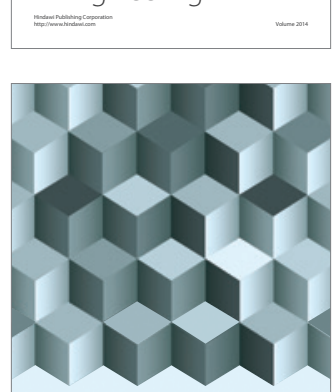

Journal of

Function Spaces
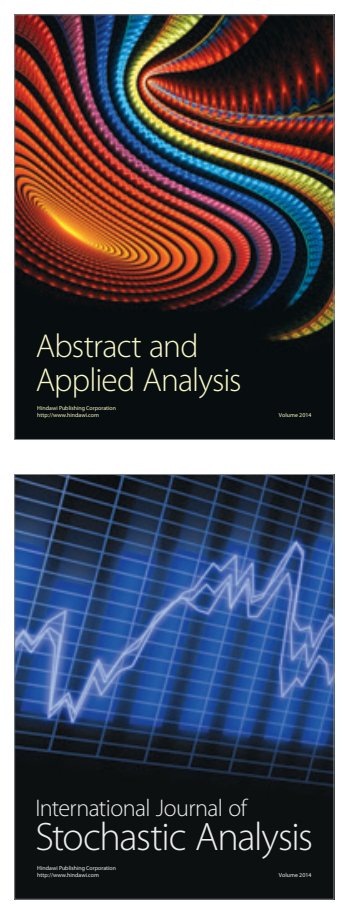

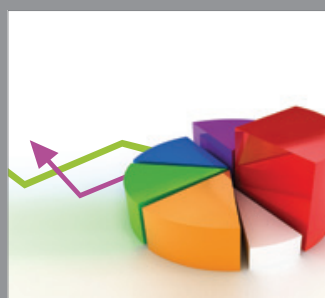

ournal of

Probability and Statistics

Promensencen
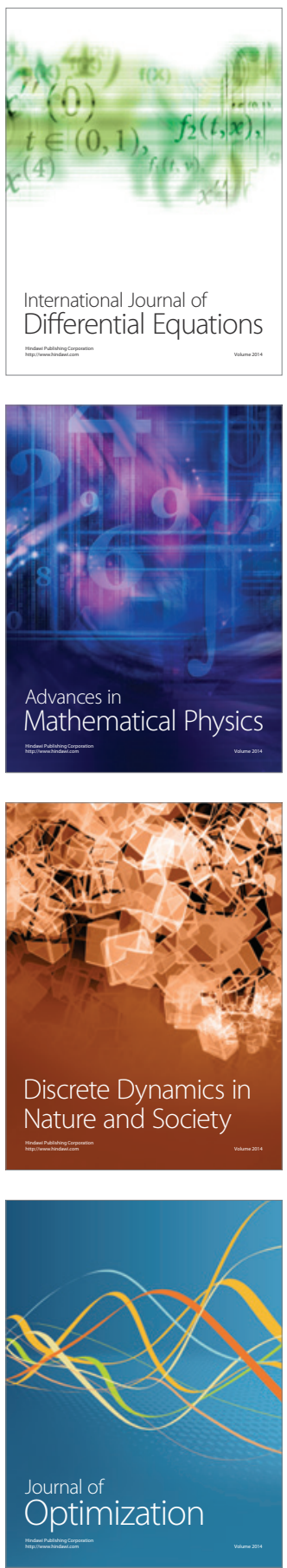